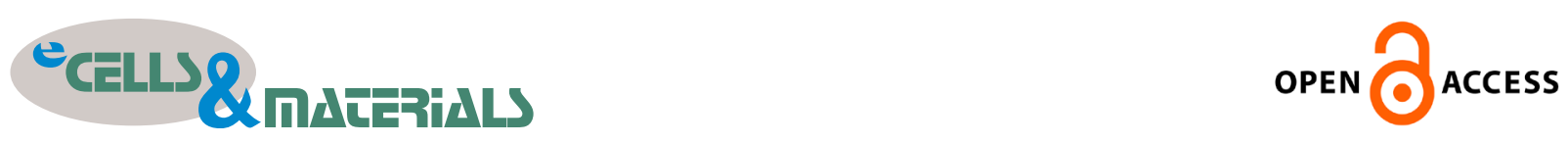

\title{
THE THERMAL CONDUCTIVITY OF CORTICAL AND CANCELLOUS BONE
}

\author{
A. Feldmann*, P. Wili, G. Maquer and P. Zysset \\ Institute for Surgical Technology and Biomechanics, University of Bern, Switzerland
}

\begin{abstract}
Surgical interventions close to vulnerable structures, such as nerves, require precise handling of surgical instruments and tools. These tools not only pose the risk of mechanical damage to soft tissues, but they also generate heat, which can lead to thermal necrosis of bone or soft tissues. Researchers and engineers are trying to improve those tools through experimentation and simulations. To simulate temperature distributions in anatomical structures, reliable material constants are needed. Therefore, this study aimed at investigating the thermal conductivity of cortical and cancellous bone. Accordingly, a custom-made steady-state experimental setup was designed and validated. 6 bovine and 3 human cortical bone samples, as well as 32 bovine cancellous bone samples, with variable bone volume fraction were tested. The cancellous bone samples were scanned by micro-computed tomography $(\mu \mathrm{CT})$ and micro-finite element $(\mu \mathrm{FE})$ voxel models were created to calculate iteratively the thermal conductivity of the bone marrow. The experimental results provided $0.64 \pm 0.04 \mathrm{~W} /$ $\mathrm{mK}$ for bovine cortical bone and $0.68 \pm 0.01 \mathrm{~W} / \mathrm{mK}$ for human cortical bone. A linear dependency of thermal conductivity on bone volume fraction was found for cancellous bone $\left[R\right.$-square $\left(R^{2}\right)=0.8096$, standard error of the estimates $(\mathrm{SEE})=0.0355 \mathrm{~W} / \mathrm{mK}$ ]. The thermal conductivity of the bone marrow was estimated to be $0.42 \pm 0.05 \mathrm{~W} / \mathrm{mK}$. These results will help to improve thermal finite element simulations of the human skeleton and aid the development of new surgical tools or procedures.
\end{abstract}

Keywords: Thermal conductivity of compact and trabecular bone, specific heat of bone, thermal bone necrosis, temperature of cutting or drilling of bone.

*Address for correspondence: Arne Feldmann, Restelbergstrasse 79, 8044 Zurich, Switzerland.

Telephone: +41 $791983941 \quad$ Email: arne.feldmann@istb.unibe.ch

\section{Introduction}

Many surgical interventions require the use of drilling or cutting tools to remove parts of the skeleton. Those tools create heat, which can lead to thermal necrosis of bone or surrounding tissues (Augustin et al., 2012; Pandey et al., 2013). Tissue damage is time- and temperature-dependent and the necrosis threshold, which is described in so-called cumulative equivalent minutes, is different for each tissue (Sapareto et al., 1984). For example, thermal bone necrosis starts at $47^{\circ} \mathrm{C}$ after $1 \mathrm{~min}$ of exposure (Eriksson et al., 1984) or at $55{ }^{\circ} \mathrm{C}$ after $30 \mathrm{~s}$ (Lundskog, 1972). Additional reasons for thermal damage are tumour ablation, cement hardening or magnetic resonance heating.

Many researchers are studying the prevention of tissue damage by optimising surgical tools or process parameters through experiments or simulations (Augustin et al., 2012; Feldmann et al., 2016a; Pandey et al., 2013). These simulations require the prior knowledge of material constants such as density, thermal conductivity or specific heat.
Cortical bone's apparent density varies from 1800 to $2100 \mathrm{~kg} / \mathrm{m}^{3}$, whereas trabecular bone's apparent density exhibits a much broader range, from 150 to $800 \mathrm{~kg} / \mathrm{m}^{3}$, depending on the anatomical site (Currey, 2006). The amount of bone within a certain region is defined as a percentage of bone volume to total volume of the defined region (bone volume/total volume $=\mathrm{BV} / \mathrm{TV})$.

The specific heat of cortical bone is $1260 \mathrm{~J} / \mathrm{kgK}$ (Huiskes et al., 1979; Lundskog, 1972). The emissivity of cortical bone, which is necessary for thermal imaging, is $\varepsilon=0.96 \pm 0.01$ (Feldmann et al., 2016b).

Previous studies show the thermal conductivity of bovine cortical bone to be between 0.2 and $12.8 \mathrm{~W} /$ mK (Biyikli et al., 1986; Davidson et al., 2000; Moses et al., 1995). The most extensive experimental study is that carried out by Davidson et al. (2000). They report the thermal conductivity of bovine cortical bone with respect to its micro structure (haversian) orientation, using a custom steady-state setup and find a slight variation from $0.53 \mathrm{~W} / \mathrm{mK}$ to $0.58 \mathrm{~W} /$ $\mathrm{mK}$ from the circumferential to longitudinal 

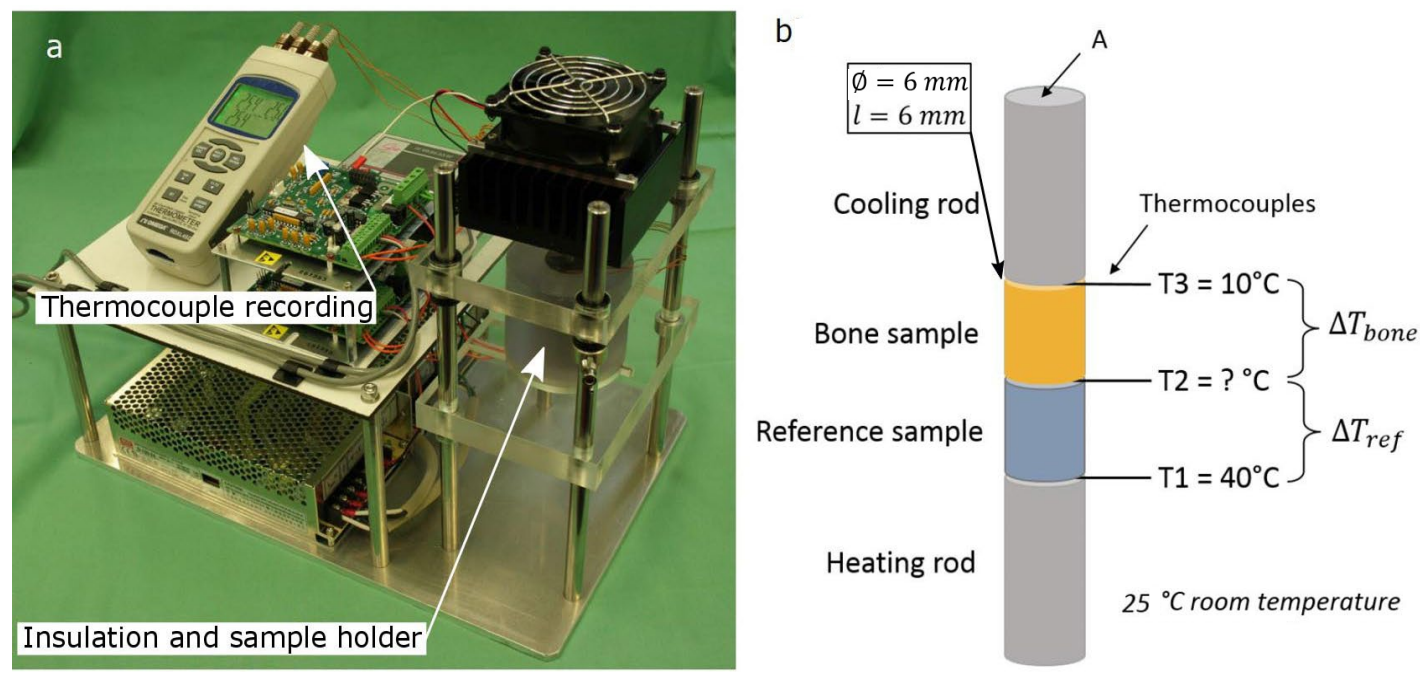

Fig. 1. (a) Whole experimental setup with thermocouple measurement device and inserted thermocouples. (b) Detailed view of samples, aluminium rods and thermocouples (normally sited to the inside of the insulation). Surrounding insulation material and sealing o-rings are not shown. Boundary temperatures T1 and T3 were kept constant, while temperature T2 depended on the thermal conductivity of the sample.

direction. Zhang et al. (2014), using the Raman shift measurement technique, confirm the range of values $(0.45-0.64 \mathrm{~W} / \mathrm{mK})$ and identify a relationship with compressive stress. The thermal conductivity first increases as a function of compressive stress, but then decreases after reaching a peak value. Only one study investigates the thermal conductivity of cancellous bone, calculating it to be around $0.3 \mathrm{~W} /$ mK (Clattenburg et al., 1975), without any information on trabecular bone microarchitecture or composition. Other researchers use finite element models (FEM) to investigate how bone cells with different thermal conductivities would experience a temperature rise in the mineralised bone matrix, but only a small temperature difference $\left(0.001{ }^{\circ} \mathrm{C}\right)$ is found between the embedded cells and the surrounding mineralised matrix (Dolan et al., 2014).

Overall, there is a lack of reliable thermal conductivity values reported for cancellous bone or human cortical bone. The aim of this study was to determine thermal conductivity of bovine and human cortical bone as well as bovine cancellous bone. Bovine samples were used due to the limited availability of human samples. The cortical bone samples were tested and compared using two different setups: a custom-made steady-state and a commercially available transient setup. Afterwards, the thermal conductivity of cancellous bone samples with different bone volume fractions was measured using the validated custom-made steady-state setup. Additionally, thermal conductivity values for bone marrow were derived from micro-finite element $(\mu \mathrm{FE})$ simulations of the cancellous bone samples.

\section{Materials and Methods}

There are different methods to measure the thermal conductivity of a material. They can be divided into steady-state and transient methods (Wakeham et al., 2000). In the steady-state setups, a constant known heat flow is assumed to stream through an object (the measurement sample). These setups are mostly designed in a so-called parallel plate arrangement, to create a temperature flow through the sample and a reference sample with a known thermal conductivity. The reference sample is needed to calculate the heat flux. Sometimes these types of measurement systems are realised with a heat flow sensor instead. The calculation of the thermal conductivity is very simple if no (lateral) heat loss and perfect heat transmission between the samples are assumed (eq. 1):

$$
\dot{q}_{\text {bone }}=\dot{q}_{\text {reference }}=\frac{k A}{l} \cdot \Delta T
$$

with " $\dot{q}$ " being the heat rate $(\mathrm{W})$, " $k$ " the thermal conductivity (W/mk), " $A$ " the cross-sectional area $\left(\mathrm{m}^{2}\right)$ and " $l$ " the length $(\mathrm{m})$ of the object (sample) (Incropera et al., 1996). The temperature difference between the two ends is denoted with " $\Delta T$ " and the temperature drop is assumed to be linear within a homogenous sample. If the material and reference sample have the same dimensions, eq. 1 can be simplified and written for the thermal conductivity of the tested material (eq. 2):

$$
k_{\text {bone }}=k_{\text {ref }} \cdot \frac{\Delta T_{\text {ref }}}{\Delta T_{\text {bone }}}
$$

In the transient method, the calculation of the thermal conductivity is more complex and based on the temporal behaviour of the temperature change of a heated sensor that is placed within the material (Wakeham et al., 2000).

In this study, both methods were used to evaluate the thermal conductivity of cortical bone, whereas only the steady-state method was used to measure 
the thermal conductivity of cancellous bone. In fact, the transient method is only able to assess homogenous samples, a condition that is not verified for trabecular bone samples. While a custom setup was designed and manufactured for the steady-state measurements, a commercial system (TPS 500; Hot Disk Instruments, Gothenburg, Sweden) was used for the transient measurements.

\section{Experimental setup \\ Steady-state setup}

The steady-state parallel plate system was designed according to the literature (Davidson et al., 2000) and based on ASTM standards (Web ref. 1). Fig. 1 shows the final system with a detailed view of the size and arrangement of samples and insulation. Pilot experiments and simulations were used to determine the sample dimensions (diameter $=\varnothing=6 \mathrm{~mm}$, length $=6 \mathrm{~mm}$ ). The optimal size was determined based on the need for a sufficient volume of bone material and the necessary reduction of lateral heat loss (a longer sample has a higher lateral surface area).

The (lateral) heat loss is a general problem of this kind of setup. Therefore, a low conductive closed foam polyethylene material was used that should, additionally to the thermal insulation, prevent water loss from the bone sample. The heat flow through the sample was realised with two aluminium rods of the same diameter that had o-rings for the same heat-sealing purpose. The aluminium rods were heated or cooled by Peltier modules (VT-127-1.01.3-71; TE Technology Inc., Traverse City, MI, USA). The temperatures of the rods were set and controlled so that the temperature at the boundaries of the samples were $40{ }^{\circ} \mathrm{C}$ and $10^{\circ} \mathrm{C}$, respectively (Fig. $1 \mathbf{b})$. These temperatures were determined by pilot simulations to minimise the temperature difference to room temperature $\left(25^{\circ} \mathrm{C} \pm 15^{\circ} \mathrm{C}\right)$ and to reduce the asymmetry of heat loss between bone and reference sample.

A reference sample with the known thermal conductivity of $0.95 \mathrm{~W} / \mathrm{mK}$ (PTFE660; AngstPfister,
Zurich, Switzerland) was used. Three thermocouples type T (5SRTC-TT-TI-30-1M, error $\pm 0.5^{\circ} \mathrm{C}$; Omega, Norwalk, CT, USA) were inserted and fixed inbetween aluminium rods and samples and inbetween the reference sample and the bone sample. A sufficient amount of thermal conductive paste was added to ensure a proper heat flow in the axial direction.

Pilot tests with two reference samples were used to evaluate the remaining heat loss. To compensate this loss, a simple FE model (Abaqus 6.11; Dassault Systems, Vélizy-Villacoublay, France) was created, which represented the basic cylindrical parts of the setup: bone and reference sample, as well as the surrounding insulation material. The thermal conductivity of the surrounding insulation was $0.0245 \mathrm{~W} / \mathrm{mK}$. The reference sample components, bone sample and surrounding insulation, were modelled as cylinders with direct contact. The room temperature was $25^{\circ} \mathrm{C}$ and the temperatures of the cooling and the heating rod were $10{ }^{\circ} \mathrm{C}$ and $40{ }^{\circ} \mathrm{C}$, respectively, as shown in Fig. 1. All elements were standard linear heat transfer elements (DC3D). The model was used post-experimental and the actual thermal conductivity of all bone samples was determined by iteratively adjusting the thermal conductivity of the bone so that the temperature $\mathrm{T}_{2}$ of the simulation matched the experimental temperature. The adjusted conductivity values were slightly lower than the measured (and calculated with eq. 2 ) experimental values (in average: $\approx 0.02 \mathrm{~W} / \mathrm{mK}$ ).

\section{Transient setup}

To further validate the experimental setup, measurements of cortical bone samples were also conducted with a Hot Disk System (TPS 500, error $<5 \%$ and reproducibility $2 \%$; Hot Disk Instruments). This system uses a transient method to calculate the thermal conductivity (and specific heat). It measures a pair of samples with the advantage of a short measurement time (s), but is not able to measure heterogeneous samples. Therefore, it was not used to measure cancellous bone samples.

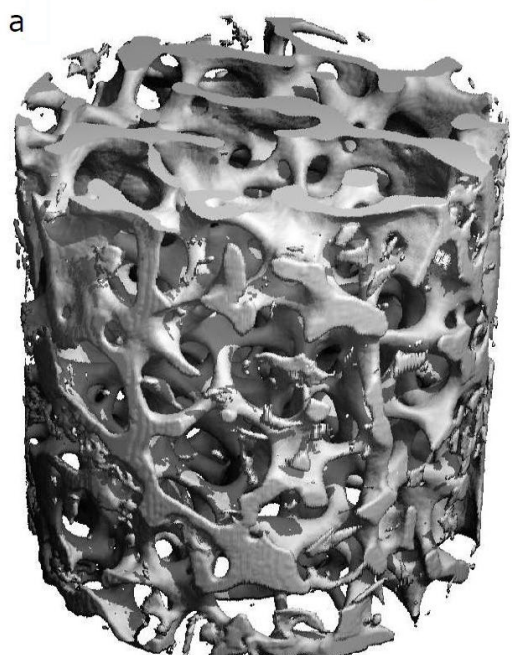

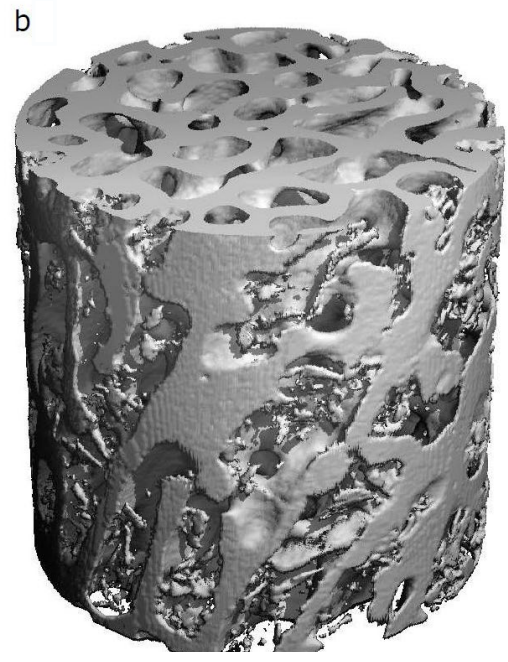

Fig. 2. $\mu \mathrm{CT}$ image of (a) low density cancellous bone sample (BV/ $\mathrm{TV}=0.2070)$ and $(\mathbf{b})$ cancellous bone sample with higher bone volume fraction $(\mathrm{BV} / \mathrm{TV}=0.3716)$. 


\section{Sample preparation}

Bovine samples were extracted from fresh-frozen $\left(-20{ }^{\circ} \mathrm{C}\right)$ tibiae of 4 -year old milk cows. The cortical samples were harvested from the diaphysis and cancellous samples from the proximal tibia beneath the tibial plateau. The human cortical samples were also fresh-frozen and obtained from the diaphysis of a femur of a 105-year old female cadaver. The $e x$ vivo human bone samples were acquired and used according to the ethics rules and approval of the Anatomy Institute, University of Bern, Switzerland. All samples were cut based on the anatomical orientation so that the main axis was along the osteonal (for cortical) or main trabecular direction. In total, 10 bovine and 3 human cortical, as well as 32 cancellous bone samples, were extracted. Additionally, 6 bovine cortical bone samples were prepared $(10 \mathrm{~mm} \times 10 \mathrm{~mm} \times 10 \mathrm{~mm})$ and used as a reference measurement when using the commercially available Hot Disk system (Hot Disk Instruments).

After pre-cutting the bones with a hand saw, the cylindrical samples were obtained using a hollow diamond core drill bit with inner diameter of $6 \mathrm{~mm}$. A computerised numerical control (CNC)-machine with very low feed rate $(0.05 \mathrm{~mm} / \mathrm{s} ; 2500 \mathrm{RPM})$ was used and samples were drilled in intervals within a water bath, to prevent excessive temperature rise.
Finally, the samples were cut to the correct length $(6 \mathrm{~mm})$ using a diamond blade band saw (EXAKT Advanced Technologies GmbH, Frankfurt am Main, Germany).

$\mu \mathrm{CT}$ images of the cancellous bone samples were taken with a $16 \mu \mathrm{m}$ resolution $(\mu \mathrm{CT} 40$; Scanco Medical, Brüttisellen, Switzerland) and morphological parameters, such as bone volume fraction (BV/TV), connectivity density (Conn.D), trabecular thickness (Tb.Th_mean and Tb.Th_SD), structure model index (SMI) and normalised mean intercept length along the axis of the sample, were extracted with the commercial Scanco Software (Harrigan et al., 1983). Fig. 2 shows an example of a cancellous bone sample with a low and a high bone volume fraction. The samples were thawed in saline solution prior to the experiments. Each experiment was run for $2 \mathrm{~h}$ to ensure temperature equilibrium. Steady-state was reached after around $90 \mathrm{~min}$ and temperatures were averaged over the following $30 \mathrm{~min}$.

\section{Micro-finite element model}

Besides using the previously mentioned FEM for improving the experimental setup, an additional $\mu F E$ model was developed to analyse the cancellous bone samples. The aim of this model was to calculate the thermal conductivity of the bone marrow using

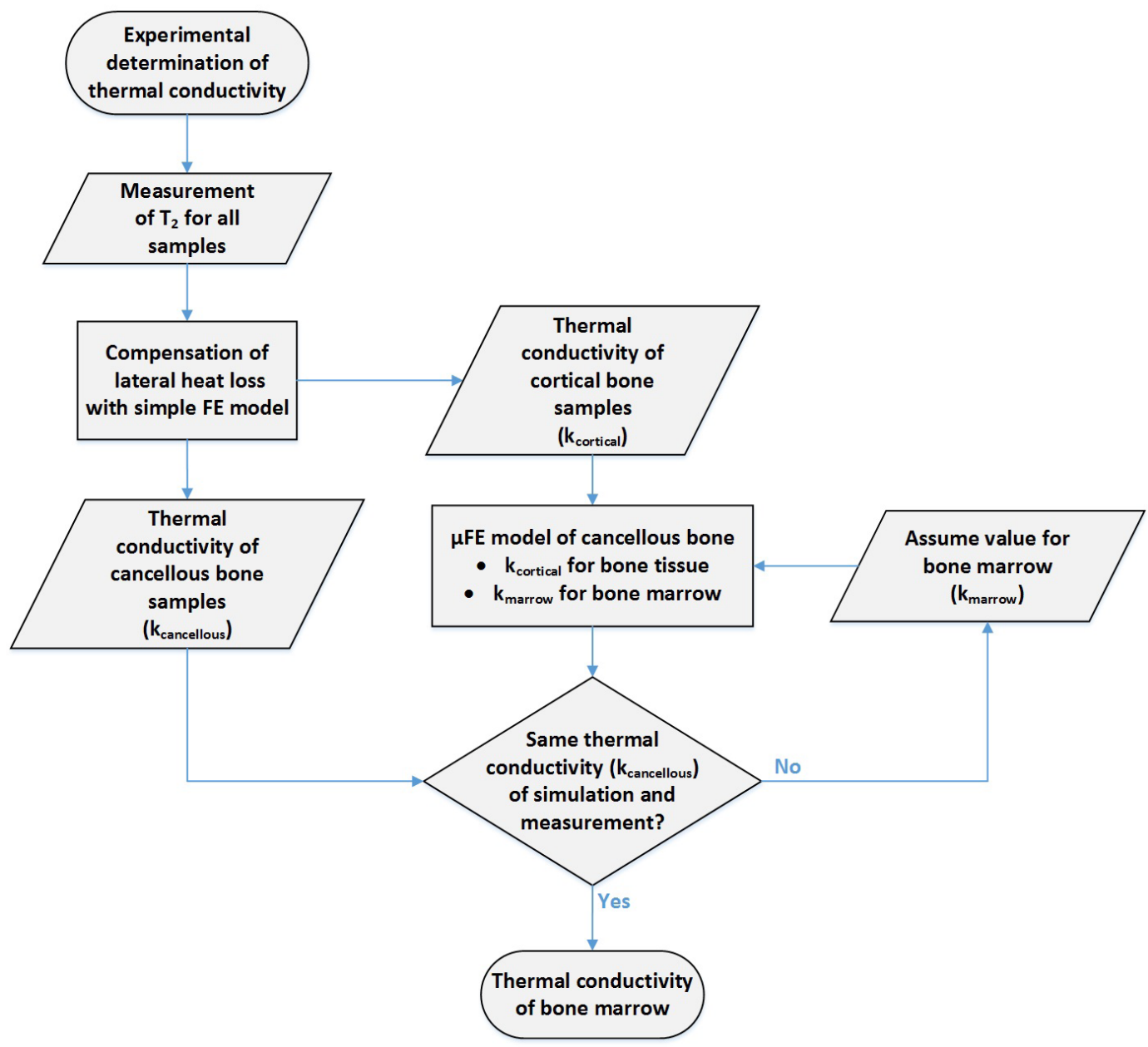

Fig. 3. Flow chart of process to inversely determine the thermal conductivity of bone marrow from the cancellous bone samples. 
an inverse method. For this calculation, the found thermal conductivity of cortical bone was used for the trabecular bone tissue. Afterwards, the thermal conductivity of the bone marrow was iteratively determined ( $\approx 5-10$ iterations) by matching the total thermal conductivity of each sample of the $\mu F E$ simulation with the previously experimentally determined total homogenised thermal conductivity of cancellous bone samples.

At this purpose, $\mu \mathrm{FE}$ voxel models were created from the coarsened $\mu \mathrm{CT}$ images $(48 \mu \mathrm{m})$ of each cancellous bone sample. Medtool 3.8 (Dr Pahr Eur-Ing., Pfaffstätten, Austria) was used for image processing (segmentation, mesh generation) and, subsequently, the model was solved with ABAQUS 6.11 (Simulia, Dassault Systèmes, Vélizy-Villacoublay, France). Boundary conditions were used to match again the experimental condition, but this time assuming no lateral heat loss (adiabatic lateral boundary conditions), because it had already been compensated for with the first FE simulation. A part of the reference sample was included in the final mesh, which had 2.1 million elements (type: DC3D8: 8-node linear brick heat transfer element), to create a heat flow and a read-out temperature $T_{2}$ between the two samples.

\section{Statistical analysis}

Two statistical analyses were carried out to evaluate the thermal conductivity of cortical and cancellous bone. Wilcoxon rank-sum test (RStudio 3.2.1; RStudio Inc., Boston, MA, USA) was used to evaluate the differences in thermal conductivity of bovine cortical bones measured with the two setups (commercial Hot Disk vs. custom steady-state) and to compare the thermal conductivity values obtained for human and bovine cortical bones using the validated steady-state setup (Fig. 3). Results with $p<0.05$ were considered statistically significant. Next, the measurements of the cancellous bone were analysed in relation to the thermal conductivity values of bovine cortical bone and to the inversely calculated bone marrow values. For this, a linear regression analysis was carried out to establish the relationship between $\mathrm{BV} / \mathrm{TV}$ and thermal conductivity (Fig. 4). Further, it was assessed if fabric (in terms of mean intercept length) or other morphometric parameters would improve the prediction of thermal conductivity, when added to $\mathrm{BV} / \mathrm{TV}$ in the form of a multilinear model.

\section{Results}

Fig. 4 shows the thermal conductivity values of bovine and human cortical bone measured with the commercially available Hot Disk and the custom made steady-state setup. The top and bottom lines of the rectangle represent the 3rd and 1st quartiles, the line in the middle the median. The top/bottom whisker denotes the maximum/minimum value or the $3 \mathrm{rd} / 1$ st quartile \pm 1.5 times the interquartile range, whichever is smaller/larger. Wilcoxon rank-sum tests showed small, although statistically significant differences ( $p=0.01827)$ between the two setups, but not between the human and bovine cortical bone samples $(p=0.07231)$. The found mean values and standard deviations were $0.60 \pm 0.01 \mathrm{~W} / \mathrm{mK}$ (bovine bone with Hot Disk), $0.64 \pm 0.04 \mathrm{~W} / \mathrm{mK}$ (bovine bone with steady-state setup) and $0.68 \pm 0.01 \mathrm{~W} / \mathrm{mK}$ (human bone with steady-state setup). All thermal conductivity results of the steady-state setup were optimised with the first FEM model, as described in the experimental setup section. Reproducibility experiments were conducted with the reference sample and a reproducibility precision of $4.88 \%$ was calculated (Gluer et al., 1995).

The total conductivity of cancellous and cortical bone samples (of the steady-state setup) was plotted dependent on bone volume fraction (Fig. 5). Additionally, the bone marrow values, which were calculated from the $\mu \mathrm{FE}$ model, are shown as blue triangles (Fig. 5). The mean and standard deviation of the thermal conductivity were $0.42 \pm 0.05 \mathrm{~W} / \mathrm{mK}$. The regression analysis for cancellous and cortical bone samples (with BV/TV assumed to be equal to 0.95 for all cortical specimens) provided an adjusted R-square

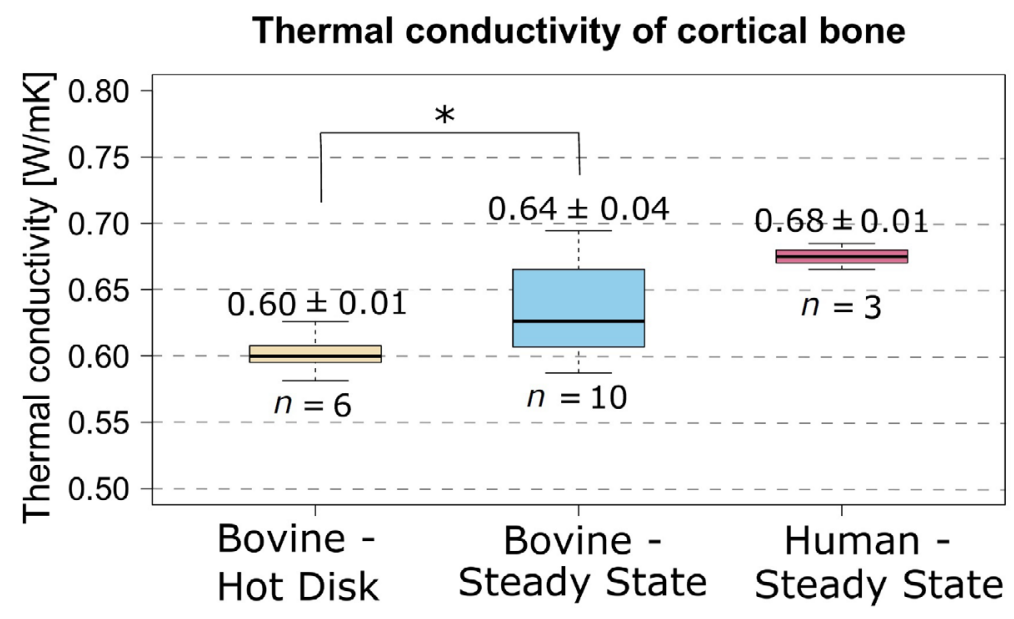

Fig. 4. Results of thermal conductivity of bovine and human cortical bone. Bovine cortical bone was measured with the custom-made steady-state setup and the commercially available Hot Disk system. Wilcoxon ranksum test showed small, although statistically significant differences ( $p=0.01827$ ) between the two setups, but not between the human and bovine cortical bone samples $(p=0.07231)$. The mean values and standard deviations are given above each boxplot. 
$\left(R^{2}\right)$ of 0.814 and a standard error of the estimates (SEE) of $0.035 \mathrm{~W} / \mathrm{mK}$ (Fig. 5). The regression line was extrapolated towards the calculated bone marrow values. The $\mathrm{R}^{2}$ was reduced for the cancellous bone samples $\left(R^{2}=0.5163, \mathrm{SEE}=0.0318 \mathrm{~W} / \mathrm{mK}\right)$. Due to the non-uniform distribution of the data, an additional Kendall's Tau test was performed with significant results (tau $=0.692$, 2-sided, $p=<2.22 \mathrm{e}-16$ ).

Fig. 6 shows the heat flux per unit area with and without the bone marrow elements. All FEM were run with the bone marrow phase being present and the bone marrow elements were only removed for a better visualisation. The heat flux was higher (red) for the trabeculae aligned along the main axis. Fig. $6 \mathbf{b}$ includes the bone marrow, which was apparently less conductive, with the heat flowing mainly through the trabeculae. Although the measured samples were all extracted along the anatomical main axis, the range in axial normalised mean intercept length was substantial (0.7-1.5), but did not correlate with the thermal conductivity. The available samples might include horizontal remnants of growth plate and might not be best suited for a detailed investigation of the influence of fabric on thermal conductivity. In the multiple regression model for predicting the thermal conductivity, the inclusion of further morphometric parameters in addition to $\mathrm{BV} / \mathrm{TV}$ did not increase the determination coefficient, compared to BV/TV alone $\left(\mathrm{R}_{\text {Conn.D }}^{2}=0.25, \mathrm{R}_{\text {SMI }}^{2}=0.32, \mathrm{R}_{\text {Tb.Th_Mean }}^{2}=0.01\right.$, $\left.\mathrm{R}_{\text {Tb.Th_SD }}^{2}=0.1\right)$. Additionally, the Hot Disk system measured the specific heat as $1216 \pm 60 \mathrm{~J} / \mathrm{kgK}$ (for assumed $\mathrm{Q}=1900 \mathrm{~kg} / \mathrm{m}^{3}$ ).

\section{Discussion}

Two different measurement techniques were used to determine the thermal conductivity of cortical and cancellous bones. Both setup and measurement techniques had advantages and disadvantages: the measurement with the transient method (Hot Disk system) was quick, but not able to measure heterogeneous materials. The custom-made steadystate setup was able to measure those samples, but needed a longer measuring time and was very sensitive to heat loss. Therefore, the system was improved and compared to the ones reported in literature with different measures: proper insulation, symmetric heat flow, sealing- and heat-lossaccounting FEM simulations.

The comparison of the thermal conductivity of cortical bone between the two measurement techniques and the literature value showed good agreement $(\approx 0.05 \mathrm{~W} / \mathrm{mK}$ difference). A small water loss could not be ruled out for the steady-state setup. However, the short measurement time and the similarity of the obtained values with the Hot Disk system suggested an appropriate sealing. The small differences between the two setups could be explained by the more difficult manual sample handling for the steady-state setup, with a reproducibility error of around $5 \%$. The reason for this could be that application of the thermal conductive paste could vary and remaining small air cavities or misalignments could change the recorded thermal conductivity values. However, the measured value for the specific heat was similar to the value reported in literature (1260 J/kgK) (Lundskog, 1972) and, therefore, a further indicator that the measured conductivity values were correct. The measured value of thermal conductivity for cortical bone was close to that of water $(\approx 0.6 \mathrm{~W} / \mathrm{mK})$. This confirmed the validity of the designed experimental setup, allowing further tests on human cortical bone, with values $(0.68 \pm 0.01 \mathrm{~W} / \mathrm{mK})$ found slightly higher than bovine bone $(0.64 \pm 0.04 \mathrm{~W} / \mathrm{mK})$.

However, the three human samples were obtained from the femoral diaphysis of a 105-year old female, which might have some structural and compositional differences compared to samples from younger individuals. This is a clear limitation of this experiment and further measurements will have to be taken on human bone samples of different age and anatomical sites to obtain more accurate values.

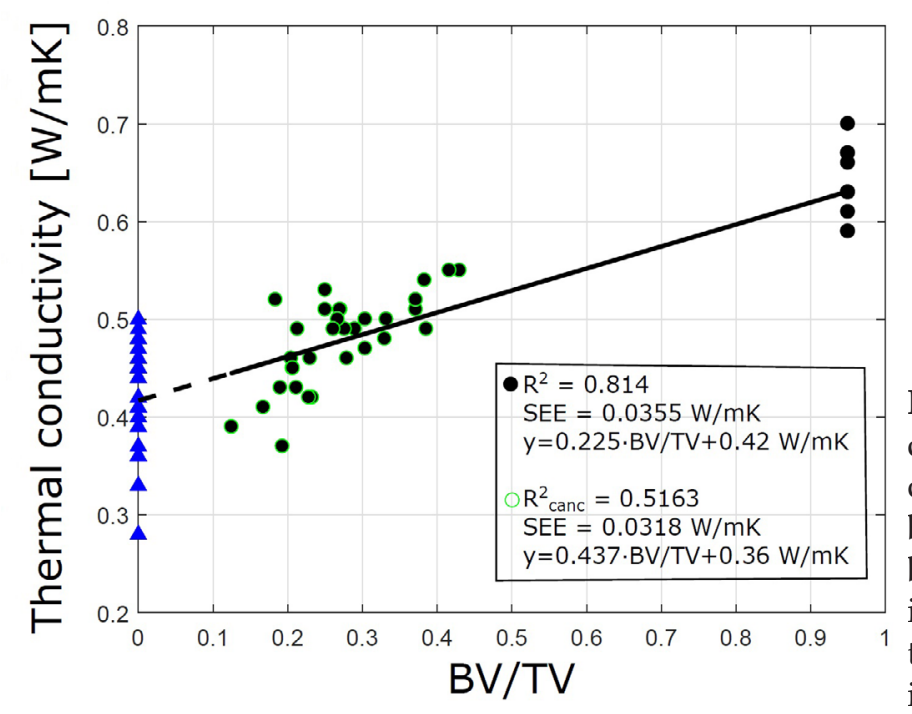

Fig. 5. Regression analysis (for cortical and cancellous bone) shows a bone volume fraction dependency of the thermal conductivity of the bovine cancellous bone samples. Bovine cortical bone samples are shown with $\mathrm{BV} / \mathrm{TV}=0.95$. The inversely calculated bone marrow (blue triangles) thermal conductivity with $\mathrm{BV} / \mathrm{TV}=0$ matches the interpolation of the regression line. 


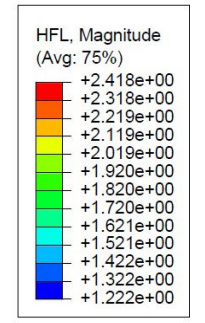

a

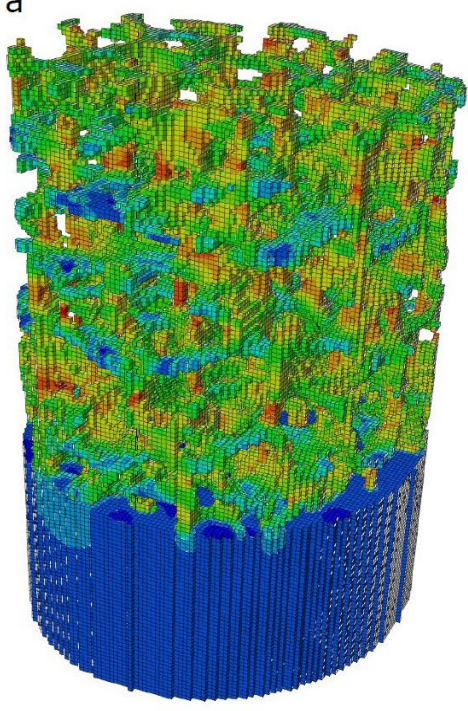

b

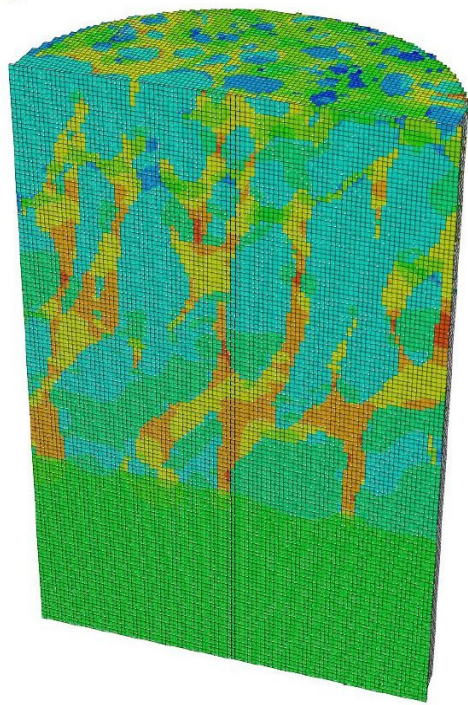

Fig. 6. Heat flux per unit area (HFL) of a cancellous bone sample (a) without bone marrow (removed for visualisation purposes) and (b) with bone marrow. The heat flux appeared to be higher (red) for the trabeculae aligned along the main axis. Also, the heat flux through the bone marrow was lower.
The steady-state setup was used to investigate the thermal conductivity of cancellous bone. The statistically significant regression with the bone volume fraction $\left(R^{2}=0.8095\right)$ suggested a linear relationship between thermal conductivity and $\mathrm{BV} /$ TV (cancellous and cortical bone). This was supported by the non-parametric Kendall Tau's test. However, more bone samples need to be tested to further study the relationship with the actual porosity of cortical bone measured by $\mu \mathrm{CT}$. It should be noted that the volumetric density of bovine cortical bone was not measured and that the correlation would slightly change when using exact BV/TV values. The lower $\mathrm{R}^{2}$ for cancellous bone was possibly due to the smaller range of $\mathrm{BV} / \mathrm{TV}$, the challenging handling of porous bone samples with the maintaining of the bone marrow in the pore and the necessary application of thermal conductive paste on the porous surface. Another reason could be the influence of the fabric, which, however, was found to be not significant in this study (multiple regression analysis). Nonetheless, the heat flux analysis suggested that there might be a dependence of thermal conductivity on trabecular orientation. If trabeculae and bone marrow would be aligned perpendicular to the heat flow, the samples would have most likely a smaller conductivity. In general, it was speculated that the thermal conductivity depended on both bone volume fraction and fabric analogy to the mechanical properties of cancellous bone (Maquer et al., 2015), but this remains to be demonstrated in future research.

Due to the measured thermal conductivity of cortical bone and the measured conductivity values of each sample, it was possible to calculate the thermal conductivity of the bone marrow with a $\mu \mathrm{FE}$ voxel model. The found conductivity of the bone marrow $(0.42 \pm 0.05 \mathrm{~W} / \mathrm{mK})$ was higher than reported in literature (0.23-0.35 W/mK) (Poppendiek et al., 1966), but matched the extrapolation of the regression line of BV/TV and thermal conductivity. The difference from the literature values was most likely due to the differences in composition between yellow and red bone marrow, which was from the proximal tibia in the cancellous bone samples. The published literature values are from yellow bone marrow, which has a lower percentage of water (= higher percentage of fat) compared to the red bone marrow and, therefore, a lower thermal conductivity [red: $0.28 \mathrm{~W} / \mathrm{mK}, 40 \%$ water content; yellow: $0.21 \mathrm{~W} / \mathrm{mK}, 15 \%$ water content (Mcintosh et al., 2010)].

The found relationships between bone volume fraction and thermal conductivity was in agreement with the mixture rule suggested by Poppendiek et al. (1969). They suggest that the thermal conductivity of any biological tissue can be calculated with respect to its composition using a parallel addition (such as electrical resistances) of the basic thermal conductivities of water, fat, collagen and air. They also show that soft tissue specimens exposed to freeze-thaw conditions can have a $10-20 \%$ higher thermal conductivity. However, this effect is possibly less relevant for hard tissues.

In general, the thermal conductivity of biological tissues is quite low, which is necessary to regulate the body temperature and protect the important organs. However, this leads also to an accumulation of the heat in a small region around the heat source, which can lead to thermal cell necrosis. While the cell necrosis of nerve tissue can be permanent, bone cell necrosis seems to be reversible. Dolan et al. (2015) show that osteocyte thermal damage (apoptosis) initiates a bone remodelling cascade. However, the effects of osteonecrosis on implant fixation (e.g. due to pre-implant drilling) is less well understood. A necrotic zone around the implant site drilling can possibly slow down osseointegration and, therefore, reduce secondary implant stability. This is shown for dental implants (Trisi et al., 2014). However, to our knowledge, there are no conclusive studies concerning this topic for orthopaedic surgeries and in vivo studies of implant stability are warranted.

It should also be noted that, besides thermal 
conductivity, the specific heat of a material is important in order to calculate the thermal diffusivity, which is needed to evaluate the rate of heat dissipation. While the specific heat of cortical bone was evaluated in this study, the specific heat of cancellous bone has to be determined in future studies. However, the values for thermal conductivity found herein might already aid the improvement of simulations, which is especially important for investigating the thermal effect of cutting tools close to nerves and other vulnerable structures. A more detailed study of the dependency of cancellous bone thermal conductivity on fabric orientation, and in particular in human bone samples, is planned.

\section{Conclusion}

This study investigated the thermal conductivity of cortical and cancellous bone. A custom made steadystate setup was improved from previous setups and validated with a commercially available setup. The thermal conductivity of bovine cortical bone and human cortical bone was found to be $0.64 \pm 0.04 \mathrm{~W} /$ $\mathrm{mK}$ and $0.68 \pm 0.01 \mathrm{~W} / \mathrm{mK}$, respectively.

32 cancellous bone samples were measured and a dependency of thermal conductivity on bone volume fraction was found. The linear regression analysis showed a significant linear relationship $\left(\mathrm{R}^{2}=0.8095\right)$, which allows for a simple calculation of the thermal conductivity with known BV/TV. Additionally, a $\mu \mathrm{FE}$ voxel model was created from $\mu \mathrm{CT}$ scans of cancellous bone samples in order to calculate the thermal conductivity of the bone marrow $(0.42 \pm 0.05 \mathrm{~W} / \mathrm{mK})$ with an inverse method. Further research is needed to assess human bone from different anatomical sites, also in relation to age and to investigate the fabric dependency of the thermal conductivity. These results might help future researchers to improve their models and to prevent bone or soft tissue necrosis in surgical procedures.

\section{Acknowledgment}

The authors would like to thank Alejandro Juarez for his contribution to the experimental setup, JuEun Lee for her pilot simulations to reduce the influence of heat loss and Thomas Rupp for his help with the measurements of the Hot Disk system at the Interstaatliche Hochschule für Technik Buchs NTB (Buchs SG, Switzerland). Additionally, we would like to acknowledge Nano Tera and the Swiss National Science Foundation for funding the research within the Hear Restore project (RTD 2013). The ex vivo human bone samples were acquired and used according to the ethics rules and approval of the Anatomy Institute, University of Bern, Switzerland. We declare no conflict of interest.

\section{References}

Augustin G, Zigman T, Davila S, Udilljak T, Staroveski T, Brezak D, Babic C (2012) Cortical bone drilling and thermal osteonecrosis. Clin Biomech (Bristol, Avon) 27: 313-325.

Biyikli S, Modest MF, Tarr R (1986) Measurements of thermal properties for human femora. J Biomed Mater Res 20: 1335-1345.

Clattenburg R, Cohen J (1975) Thermal properties of cancellous bone. J Biomed Mater Res 9: 169-182.

Currey JD (2006) Bones: structure and mechanics. Princeton University Press, Princeton: 1-436.

Davidson SR, James DF (2000) Measurement of thermal conductivity of bovine cortical bone. Med Eng Phys 22: 741-747.

Dolan EB, Vaughan TJ, Niebur GL, Casey C, Tallon D, McNamara LM (2014) How bone tissue and cells experience elevated temperatures during orthopedic cutting: anexperimental and computational investigation. J Biomech Eng 136:1-9.

Dolan EB, Haugh MG, Voisin MC, Tallon D, McNamara LM (2015) Thermally induced osteocyte damage initiates a remodelling signaling cascade. Plos One 10: e0119652.

Eriksson R, Albrektsson T (1984) The effect of heat on bone regeneration: an experimental study in the rabbit using the bone growth chamber. J Oral Maxillofac Surg 42: 705-711.

Feldmann A, Anso J, Bell B, Williamson T, Gavaghan K, Gerber N, Rohrbach H, Zysset P (2016a) Temperature prediction model for bone drilling based on density distribution and in vivo experiments for minimally invasive robotic cochlear implantation. Ann Biomed Eng 44: 1576-1586.

Feldmann A, Zysset P (2016b) Experimental determination of the emissivity of bone. Med Eng Phys 38: 1136-1138.

Gluer C, Blake G, Lu Y, Blunt BA, Jergas MI (1995) Accurate assessment of precision errors: how to measure the reproducibility of bone densitometry techniques. Osteoporos Int 5: 262-270.

Harrigan T P, Mann R W (1983) Characterization of microstructural anisotropy in orthotropic materials using a second rank tensor. J Mater Sci 19: 761-767.

Huiskes HWJ (1979) Some fundamental aspects of human joint replacement. PhD thesis, Technical University of Eindhoven, Netherlands.

Incropera FP, DeWitt DP (1996) Fundamentals of heat and mass transfer. 4th Edition, John Wiley and Sons, Purdue University.

Lundskog J (1972) Heat and bone tissue: an experimental investigation of the thermal properties of bone and threshold levels for thermal injury. Scand J Plast Reconstr Surg 9: 1-80.

Maquer G, Musy SN, Wandel J, Gross T, Zysset PK (2015) Bone volume fraction and fabric anisotropy are better determinants of trabecular bone stiffness than other morphological variables. J Bone Miner Res 30: 1000-1008. 
Mcintosh LR, Anderson V (2010) A comprehensive tissue properties database provided for the thermal assessment of a human at rest. Biophys Rev Lett 5: 129-151.

Moses W, Witthaus F, Hogan H, Laster W (1995) Measurement of the thermal conductivity of cortical bone by an inverse technique. Exp Therm Fluid Sci 11: 4-39.

Pandey RK, Panda S (2013) Drilling of bone: a comprehensive review. J Clin Orthop Trauma 4: 1530.

Poppendiek H, Randall R, Breeden J, Chambers J, Murphy J (1966) Thermal conductivity measurements and predictions for biological fluids and tissues. Cryobiology 3: 318-327.

Sapareto SA, Dewey WC (1984) Thermal dose determination in cancer therapy. Int J Radiat Oncol Biol Phys 10: 787-800.

Trisi P, Beraedini M, Falco A, Vulpiani MP, Perfetti G (2014) Insufficient irrigation induces peri-implant bone resorption: an in vivo histologic analysis in sheep. Clin Oral Implants Res 25: 696-701.

Wakeham WA, Assael MJ (2000) Mechanical variables measurement - solid, fluid, and thermal. Ed. JG Webster. CRC Press, Boca Raton, FL, USA.

Zhang Y, Gan M, Tomar V (2014) Raman thermometry based thermal conductivity measurement of bovine cortical bone as a function of compressive stress. J Nanotechnol Eng Med 5: 1-11.

\section{Web References}

1. https://www.astm.org/DATABASE.CART/ HISTORICAL/C518-10.htm [21-01-18]

\section{Discussion with Reviewer}

Glen Niebur: Would the anisotropy of the thermal conductivity play a major role in applying these data to larger models?

Authors: We think that the anisotropy may have an influence on cancellous bone [and small influence on cortical (Davidson et al., 2000)], but further research is needed to prove it.

Glen Niebur: Could the transient behaviour during the experiment be used to estimate the heat capacity of the bone, perhaps using the data from the Hot Disk system as an initial estimate?

Authors: Yes, this is also topic of further research.

Glen Niebur: How can these data be applied to understand the potential for thermal necrosis during bone preparation or bone cement hardening?

Authors: The found thermal conductivity values will enable more detailed simulations (e.g. FEM) of the transmission of heat from the heat source (e.g. bone cement, drilling) into the bone (cancellous or cortical). Further investigations of the cumulative equivalent minutes (CEM47) will help to assess the damage to bone or cells of other tissues.

Editor's note: The Scientific Editor responsible for this paper was Juerg Gasser. 